\title{
Seroprevalence in Bats and Detection of Borrelia burgdorferi in Bat Ectoparasites
}

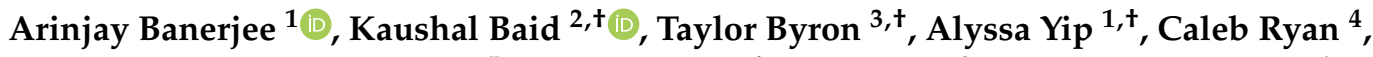 \\ Prasobh Raveendran Thampy ${ }^{5}$, Hugh Broders ${ }^{4}$, Paul Faure ${ }^{3}$ and Karen Mossman ${ }^{1, *}$ \\ 1 Michael G. DeGroote Institute for Infectious Disease Research, McMaster Immunology Research Centre, \\ Department of Pathology and Molecular Medicine, McMaster University, Hamilton, ON L8S 4L8, Canada; \\ banera9@mcmaster.ca (A.B.); alyssa.yip14@gmail.com (A.Y.) \\ 2 Department of Biochemistry and Biomedical Sciences, McMaster Immunology Research Center, \\ McMaster University, Hamilton, ON L8S 4L8, Canada; baidk@mcmaster.ca \\ 3 Department of Psychology, Neuroscience \& Behaviour, McMaster University, \\ Hamilton, ON L8S 4K1, Canada; byront@mcmaster.ca (T.B.); paul4@mcmaster.ca (P.F.) \\ 4 Department of Biology, University of Waterloo, Waterloo, ON N2L 3G1, Canada; \\ c9ryan@edu.uwaterloo.ca (C.R.); hugh.broders@uwaterloo.ca (H.B.) \\ 5 Department of Veterinary Microbiology, University of Saskatchewan, Saskatoon, SK S7N 5B4, Canada; \\ prr154@mail.usask.ca \\ * Correspondence: mossk@mcmaster.ca; Tel.: +905-525-9140 (ext. 23542) \\ + These authors contributed equally to this work.
}

Received: 8 February 2020; Accepted: 18 March 2020; Published: 20 March 2020

check for updates

\begin{abstract}
The role of bats in the enzootic cycle of Lyme disease and relapsing fever-causing bacteria is a matter of speculation. In Canada, Borrelia burgdorferi sensu stricto (ss) is the genospecies that is responsible for most cases of Lyme disease in humans. In this study, we determined if big brown bats, Eptesicus fuscus, have been exposed to spirochetes from the genus Borrelia. We collected serum from 31 bats and tested them for the presence of anti-Borrelia burgdorferi antibodies using a commercial enzyme-linked immunosorbent assay (ELISA). We detected cross-reactive antibodies to Borrelia spp. in 14 of 31 bats. We confirmed the ELISA data using a commercial immunoblot assay. Pooled sera from ELISA-positive bats also cross-reacted with Borrelia antigens coated on the immunoblot strips, whereas pooled sera from ELISA-negative bats did not bind to Borrelia spp. antigens. Furthermore, to identify if bat ectoparasites, such as mites, can carry Borrelia spp., we analyzed DNA from 142 bat ectoparasites that were collected between 2003 and 2019. We detected DNA for the Borrelia burgdorferi flaB gene in one bat mite, Spinturnix americanus. The low detection rate of Borrelia burgdorferi DNA in bat ectoparasites suggests that bats are not reservoirs of this bacterium. Data from this study also raises intriguing questions about Borrelia infections in bats, including the role of humoral immunity and the ability of bats to be infected with Borrelia burgdorferi. This study can lead to more sampling efforts and controlled laboratory studies to identify if bats can be infected with Borrelia burgdorferi and the role of bat ectoparasites, such as S. americanus, in the transmission of this spirochete. Furthermore, we outlined reagents that can be used to adapt ELISA kits and immunoblot strips for use with bat sera.
\end{abstract}

Keywords: Borrelia; big brown bats; seroprevalence; ectoparasite

\section{Introduction}

Numerous emerging pathogenic viruses have been detected in bats [1], but few studies have looked at the prevalence of bacteria that can cause disease in other mammals, including humans. Lyme disease is a tick-borne disease caused by spirochete bacteria belonging to the Borrelia burgdorferi sensu lato (sl) genospecies complex [2,3]. In North America, these tick-borne bacteria are transmitted 
to vertebrate hosts, including humans, through the bite of Ixodes ticks. In Canada, Borrelia burgdorferi sensu stricto (ss) is the genospecies that is responsible for most cases of Lyme disease in humans [4]. Other species, such as Borrelia bissettii, Borrelia kurtenbachii, and Borrelia mayonii, are less commonly associated with human disease.

Rodents and birds are the reservoir hosts that support the enzootic cycle of $B$. burgdorferi sl in nature [5,6]. In addition to the B. burgdorferi sl group, there are other species of Borrelia that can cause relapsing fever [7]. Although the role of bats as reservoirs of relapsing fever-causing bacteria was speculated upon [8] and sequences of Borrelia spp. were identified in bat ectoparasites [9-12], a limited number of studies looked at the seroprevalence of B. burgdorferi in insectivorous bats, along with detecting B. burgdorferi DNA in their ectoparasites.

In this study, we sought to identify if bats in Canada were exposed to B. burgdorferi spirochetes. Furthermore, archived samples of bat ectoparasites were used as surrogates to determine if bats could potentially be exposed to B. burgdorferi via vectors.

\section{Methods}

\subsection{Sample Collection (Bats and Ectoparasites)}

Between 2012 and 2018, 31 big brown bats (Eptesicus fuscus) were collected and maintained in a captive colony (see Supplementary Table S1 for details on location, year bats were captured, and seropositivity status). All visible ectoparasites were removed and bats were treated with topical selamectin (10-20 $\mu \mathrm{L}$, Revolution ${ }^{\odot}$ purple for dogs; $120 \mathrm{mg} / \mathrm{mL}$ ) prior to introduction to the colony. Blood was collected from the interfemoral vein of healthy big brown bats $(n=31)$ and serum was separated by centrifuging the blood at $1500 \mathrm{~g}$ (Beckman Coulter, Brea, CA, USA) for $15 \mathrm{~min}$. Serum was aliquoted and frozen at $-80^{\circ} \mathrm{C}$ prior to analysis. Blood was also collected from bats that were born in captivity within this colony (Bat IDs: 13 pink, 189 white, 31 green, 8 pink, 50 green, 10 pink, 54 blue, 39 gray, 57 sky, 26 green, and 108 red) to monitor exposure within the colony. Bats born in captivity were categorized under Hamilton for location. Ectoparasites $(n=142)$ were collected from Myotis lucifugus, E. fuscus, and Myotis septentrionalis from Eastern Canada between 2003 and 2019 (see Supplementary Table S2 for demographic details of the samples, reproductive status and gender of bats, and the species of ectoparasite collected from bats). Animal protocols for bat handling were approved by McMaster University's and Saint Mary's University's animal research ethics boards. For sample details, see Supplementary Tables S1 and S2.

\subsection{Enzyme-Linked Immunosorbent Assay (ELISA)}

To detect antibodies against B. burgdorferi in E. fuscus serum, a commercial ELISA (GenWay Biotech, San Diego, CA, USA) was used. As described by the manufacturer, the ELISA plates were coated with Borrelia burgdorferi antigens. Samples were diluted 1:101 and the assay was performed following the manufacturer's recommendations. All samples were assayed in duplicate. To detect bat immunoglobulin $\mathrm{G}(\mathrm{IgG}$ ) that bound to B. burgdorferi antigens, $5 \mu \mathrm{g} / \mathrm{mL}$ polyclonal goat anti-bat IgG labelled with horseradish peroxidase was used (anti-bat IgG-HRP; Bethyl Laboratories Inc., Montgomery, TX, USA). Control human samples were detected using an anti-human HRP conjugate that was supplied with the kit (GenWay Biotech, San Diego, CA, USA). An anti-bat IgG-HRP-only control and a substrate control were included with every assay to account for non-specific absorbance. Absorbance values for bat serum samples were normalized to both the anti-bat IgG-HRP control and the substrate control. Human control samples were normalized to the substrate control as recommended by the manufacturer.

\subsection{Immunoblots}

To detect anti-Borrelia antibodies in bat (E. fuscus) sera, immunoblots were performed using commercially available strips that were coated with antigens from B. burgdorferi sensu stricto (Bb), 
Borrelia afzelii (Bf), and Borrelia garinii (Bg) (EUROIMMUN, Lubeck, Germany). For antigen description, see the manufacturer's website. The manufacturer's recommended procedure was followed for the assay, but the secondary antibodies and the detection chemistry were altered to adapt the kit for bat sera. Briefly, the strips were incubated with $1.5 \mathrm{~mL}$ of $0.1 \times$ human positive control (supplied as a $50 \times$ concentrate). A quantity of $1.5 \mathrm{~mL}$ of diluent was used (universal buffer; EUROIMMUN, Lubeck, Germany) as negative human control. For bats, sera were pooled from ELISA positive or negative bat samples (see Supplementary Table S1) in $1.5 \mathrm{~mL}$ of universal buffer. After blocking the strips in $1.5 \mathrm{~mL}$ of universal buffer for $15 \mathrm{~min}, 1.5 \mathrm{~mL}$ of the respective sera were added on the strips in $15 \mathrm{~mL}$ conical screw cap tubes. The tubes were gently rocked on a rocking platform for $1.5 \mathrm{~h}$ at room temperature. After $1.5 \mathrm{~h}$, sera were removed and the strips were washed three times in $1.5 \mathrm{~mL}$ of universal buffer for 5 min each on a rocking platform. After the washes, strips that had been incubated with human sera were incubated in a 1:10,000 dilution of goat anti-human IgG-HRP conjugate (Millipore, Burlington, MA, USA; catalogue number: AP112P) and strips that had been incubated with bat sera were incubated with a 1:5000 dilution of goat anti-bat IgG-HRP conjugate (Bethyl laboratories Inc., Montgomery, TX, USA; catalogue number: A140-118P). All antibodies were diluted in $1.5 \mathrm{~mL}$ of universal buffer. The strips were incubated in the diluted antibodies for $1 \mathrm{~h}$ at room temperature on a rocking platform. The strips were then washed three times, as mentioned above, and processed for signal development using a non-commercial enhanced chemiluminescence (ECL) solution [13].

\subsection{Bacterial Isolation and Detection}

A BSK-H media with $6 \%$ rabbit serum (Sigma-Aldrich, St. Louis, MO, USA) was used for bacterial isolation. Blood from E. fuscus bats were pooled and inoculated in two $15 \mathrm{~mL}$ tubes, each containing $6 \mathrm{~mL}$ of BSK-H media. The tubes were incubated at $33{ }^{\circ} \mathrm{C}$ with $5 \% \mathrm{CO}_{2}$ in a humidified incubator for 8 weeks. Fresh media was added to the culture every week and the cultures were sub-cultured every two weeks. Aliquots were processed for gram staining (Fischer Scientific, Waltham, MA, USA) and immunofluorescent staining using rabbit anti-Borrelia burgdorferi antibody (BioRad, Hercules, CA, USA) every two weeks. Gram staining was carried out following the manufacturer's recommendations (Millipore Sigma, Burlington, MA, USA). Immunofluorescent staining was carried out as previously mentioned [14]. For immunofluorescent staining, a 1:100 dilution of the primary antibody (rabbit anti-Borrelia burgdorferi) and a 1:400 dilution of the secondary antibody (Donkey anti-rabbit Alexa Fluor 488; Life Technologies, Carlsbad, CA, USA) were used. Slides were observed using a Zeiss fluorescent microscope. Cpn60 sequencing of the total bacterial population was performed, as previously mentioned [15].

\subsection{Polymerase Chain Reaction (PCR)}

DNA extractions were performed from ectoparasites, as previously mentioned [16]. The ectoparasites were classified by amplifying a $658 \mathrm{bp}$ fragment of the cytochrome oxidase subunit I (COI) gene using polymerase chain reaction, as previously mentioned [17]. Briefly, PCR was performed with Q5 high-fidelity DNA polymerase, using the manufacturer's recommended protocol (New England Biolabs Inc., Ipswich, MA, USA). 5 uL of DNA was used as template. Primers LCO1490: 5'-GGTCAACAAATCATAAAGATATTGG-3' and HCO2198: 5'-TAAACTTCAGGGTGACCAAAAAATCA-3' were used to amplify the COI gene. For amplification, an initial step at $98^{\circ} \mathrm{C}$ for $30 \mathrm{~s}$ was followed by 40 cycles of denaturation at $98^{\circ} \mathrm{C}$ for $10 \mathrm{~s}$, annealing at 56 ${ }^{\circ} \mathrm{C}$ for $10 \mathrm{~s}$, and elongation at $72{ }^{\circ} \mathrm{C}$ for $1 \mathrm{~min}$. Final extension was performed at $72{ }^{\circ} \mathrm{C}$ for $2 \mathrm{~min}$. DNA from Borrelia burgdorferi was detected by amplifying a $370 \mathrm{bp}$ fragment of the flaB gene using a previously published nested PCR protocol [18]. Briefly, Borrelia genus specific primers [18] were used for nested PCR. Primers used for nested PCR-1 were FO1: AAGTAGAAAAAGTCTTAGTAAGAATGAAGGA and FO2: AATTGCATACTCAGTACTAT TCTTTATAGAT. Primers used for nested PCR-2 were FI1-CACATATTCAGATGCAGA CAGAGGTTCTA and FI2: GAAGGTGCTGTAGCAGGTGCTGGCT GT. For amplification, an initial step at $94{ }^{\circ} \mathrm{C}$ for 3 min was followed by 35 cycles of denaturation at 
$94{ }^{\circ} \mathrm{C}$ for $30 \mathrm{~s}$, annealing at $50{ }^{\circ} \mathrm{C}$ for $30 \mathrm{~s}$, and elongation at $72{ }^{\circ} \mathrm{C}$ for $1 \mathrm{~min}$. Final extension was performed at $72{ }^{\circ} \mathrm{C}$ for $10 \mathrm{~min}$.

\subsection{Data Analysis}

The commercial ELISA kit contained control samples to determine human cut-off values. However, to determine the cut-off values for bat serum samples, these controls could not be utilized. We determined the mean absorbance value of negative serum samples (Bat IDs: 8 pink, 50 green, 54 blue, 39 gray, 58 sky, 97 purple, 26 green, 19 sky, 38 blue, and 88 yellow) and established a cut-off value of 3 standard deviations (i.e., a cut-off absorbance value of 0.475 ) to identify bat serum samples that were positive.

\subsection{Phylogenetic Tree}

Multiple sequence alignment and phylogenetic trees were constructed using MEGA 7 (version 7.0.26, https://www.megasoftware.net, accessed on $10^{\text {th }}$ September, 2019) [19].

\section{Results}

Eptesicus fuscus bats were collected from different areas in Ontario between 2012 and 2018 and maintained in a research colony (Figure 1A). To detect IgG in bat sera that bound to B. burgdorferi antigens, we analyzed bat sera and supplied human control sera using a commercial ELISA kit. We detected B. burgdorferi cross-reactive antibodies in 14 of 31 (45.16\%) bats (Figure 1B). Sera from 4 of $11(36.36 \%)$ bats born in captivity also tested positive (Supplementary Table S1).

Next, immunoblot analyses were performed using commercially available strips that were coated with antigens from B. burgdorferi ss, B. garinii, and B. afzelii. Pooled sera from ELISA-positive bats cross-reacted with multiple antigens, including $\mathrm{p} 41$ (flagellin), OspC, and $\mathrm{p} 18$, whereas pooled sera from ELISA-negative bats did not cross-react with antigens on the immunoblot strip (Figure 1C). Although we attempted to culture Borrelia spp. from bat blood, we were unable to detect spirochetes in the culture by immunofluorescence microscopy or cpn60 sequencing of the bacterial population.

To detect the B. burgdorferi flaB gene in bat ectoparasites (Figure 1D and Supplementary Table S2), DNA extracted from ectoparasites collected from Myotis lucifugus, E. fuscus, and Myotis septentrionalis from Eastern Canada between 2003 and 2019 were analyzed. A 370 bp fragment of the B. burgdorferi flaB gene was amplified from one bat mite, Spinturnix americanus, that was collected from Myotis lucifugus in 2011 (the sequence information has been submitted to the GenBank database under accession number MN954474) (Figure 1E and Supplementary Table S2). 


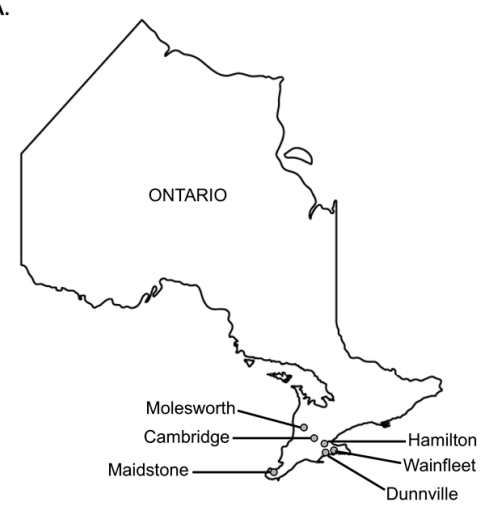

c.

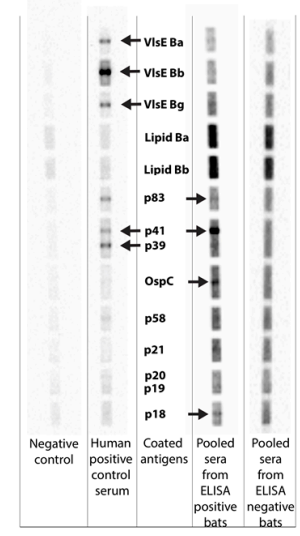

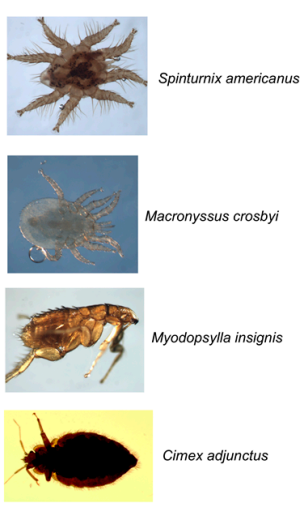

B.
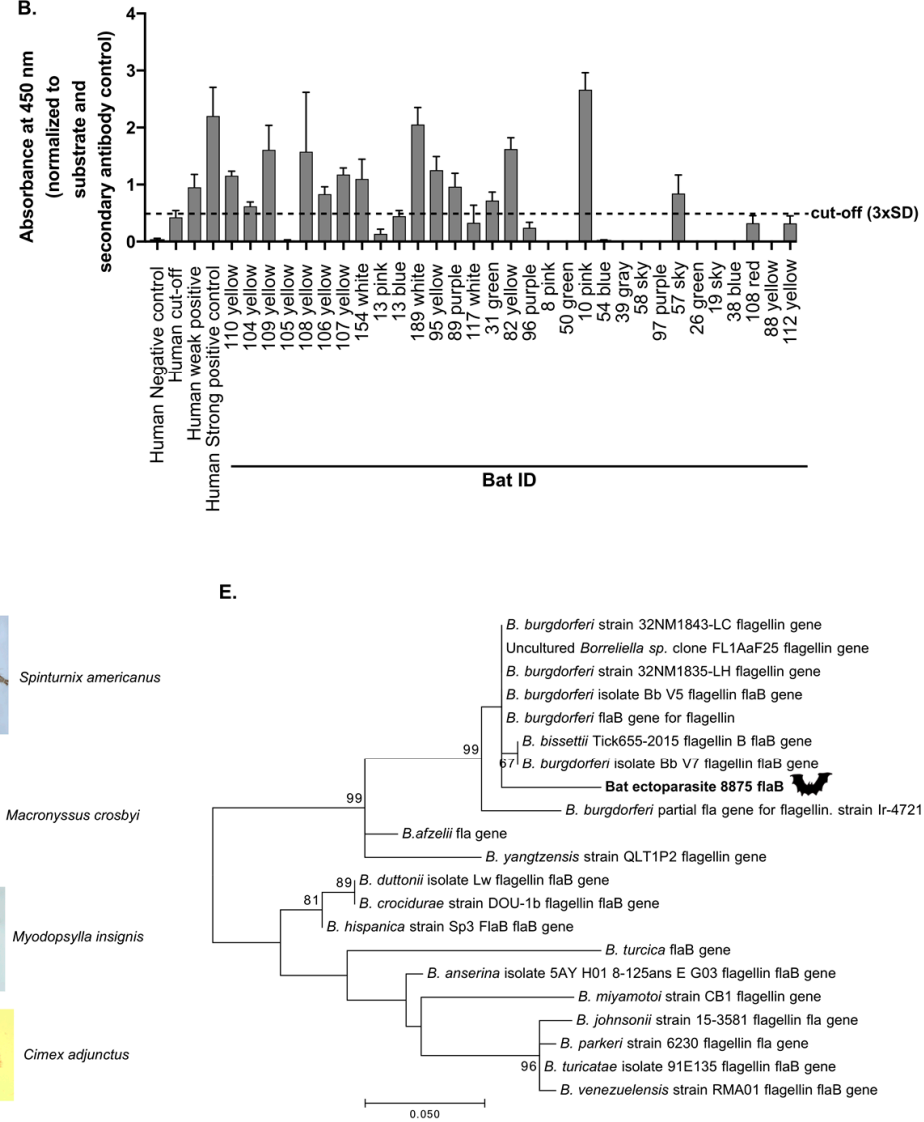

Figure 1. Detection of antibodies to Borrelia spp. in Eptesicus fuscus and the flaB gene segment in bat ectoparasites. We extracted sera from wild-caught and laboratory-bred E. fuscus to detect antibodies to Borrelia. A. Sites of bat collection. Bats were later kept in a captive research colony. B. Bar graph: antibody levels detected in E. fuscus serum samples using a commercial ELISA kit specific for anti-Borrelia burgdorferi antibodies. Human control sera were assayed in parallel. The cut-off for bat samples was set at 0.475 and is represented by the dotted line. C. Reactivity of pooled bat (E. fuscus) and control human sera with antigens from B. burgdorferi, Borrelia afzelii, and Borrelia garinii. See manufacturer's manual for antigen details (EUROIMMUN, Germany). D. Ectoparasites collected from bats in Canada. E. Detection of Borrelia burgdorferi flaB gene fragment in a bat ectoparasite. The maximum likelihood tree (1000 bootstraps) showing the phylogenetic relationship of the Borrelia burgdorferi flaB gene segment detected in 1 of 142 bat ectoparasites that were collected between 2003 and 2019 is shown. The tree is drawn to scale, with branch lengths measured by the number of substitutions per site. The percentage of trees in which the associated taxa are clustered together is shown next to the branches. Values over 60 are shown. Evolutionary analysis was conducted in MEGA7. The following terms are used: $\mathrm{SD}$ (standard deviation); $\mathrm{Ba}$ (Borrelia afzelii); $\mathrm{Bb}$ (Borrelia burgdorferi); Bg (Borrelia garinii).

\section{Discussion}

Bats are speculated to be reservoirs for several viruses [1], including tick-borne viruses [20]. Recently, the role of cave-dwelling bats and Ixodes ticks in the life cycle of B. burgdorferi sl was demonstrated in Romania and Poland [10]. Seroprevalence data from our study demonstrate that wild-caught and captive-bred Canadian bats have been exposed to B. burgdorferi or an antigenically related bacterium. Our assays were not designed to specifically detect antibodies to relapsing fever-causing spirochetes in bats; however, the presence of cross-reactive antibodies against antigenically similar spirochetes cannot be ruled out. Since wild-caught bats and bats born in captivity were either positive or negative within the same colony, it is unlikely that ectoparasites transmitted the bacterium within the research colony, but this possibility cannot be ruled out. These data raise intriguing questions about the possibility of vertical transmission of Borrelia spp. in bats, individual- or population-level 
persistence of infection, multiple exposures to Borrelia spirochetes, and the role of maternal antibodies in juvenile bats. Humoral immunity in bats is poorly understood [21]. As bat reagents and tools become available, it will be interesting to explore aspects of bat humoral immune responses against parasitizing bacteria.

Despite multiple attempts, we were unable to detect Borrelia DNA in bat blood cells using a nested PCR approach for multiple targets (16s rRNA, 5s-23s rRNA intergenic spacer, flaB gene; data not shown). In addition, we were unable to culture spirochetes from E. fuscus blood. This is not surprising, since Borrelia spirochetes are likely to be present in very low numbers in an asymptomatic host. Furthermore, blood may not be a suitable sample; more invasive sampling, including tissues, may be required to isolate any Borrelia-like spirochetes from bats.

B. burgdorferi sl was previously detected in mites from the genus Laelaps [22]. We detected a B. burgdorferi flaB gene segment in a bat mite, Spinturnix americanus. The role of S. americanus in acquiring and transmitting Borrelia spirochetes in bats needs to be investigated. From our data, the possibility that bats in Canada can acquire the spirochete cannot be ruled out. Furthermore, detecting B. burgdorferi DNA in a bat mite does not imply transmission or acquisition of the spirochete to and from bats, respectively. As discussed below, controlled laboratory experiments are required to establish the acquisition and transmission potential of B. burgdorferi by bat mites. Larger numbers of ectoparasites from bats, including E. fuscus in Ontario, need to be investigated to support our sero-surveillance data. Collecting additional samples from the sampling site of ectoparasite 8875 might allow for the identification of any localized spread of Borrelia spp. It is also possible that bats are exposed to Borrelia spp. through a different route, but do not sustain an infection for prolonged periods of time, thus limiting the opportunity for bat ectoparasites to acquire the spirochete through a blood meal. Surveillance data from bat ectoparasites can enable researchers to rationalize the development of in vivo experiments with bats and their ectoparasites in order to fully dissect the ability of bat mites to acquire and transmit $B$. burgdorferi within bat populations and to other mammalian species, such as rodents.

In our attempt to identify cross-reactive Borrelia burgdorferi antibodies in bats, we optimized and developed methods to adapt existing commercial ELISA kits and immunoblot strips for use with bat sera. These techniques and information about anti-bat secondary antibodies can allow other researchers to adapt and utilize commercial kits for the study of bat sera.

Bats play an important ecological role and carry out vital functions, such as pollination, insect population control, and seed dispersal. It is important to study and reduce human-bat interactions to prevent bat population declines due to human activities, while also mitigating risks of pathogen spillover from bats to humans.

Supplementary Materials: The following are available online at http://www.mdpi.com/2076-2607/8/3/440/s1, Table S1: Location, year bats were captured, and seropositivity status of sera collected from bats. Table S2: Demographic details of ectoparasite samples, species, reproductive status and gender of bats, and the species of ectoparasite collected from bats.

Author Contributions: Conceptualization, A.B. and K.M.; Methodology, A.B., K.B., C.R., and P.R.T.; Software, A.B., C.R., and P.R.T.; Validation, A.B., K.B., and P.R.T.; Formal Analysis, A.B., K.B., and P.R.T.; Investigation, A.B., K.B., T.B., A.Y., C.R., P.R.T., and H.B.; Resources, T.B., P.F., and H.B.; Data Curation, A.B., K.B., C.R., T.B., and P.R.T.; Writing-Original Draft Preparation, A.B.; Writing-Review \& Editing, A.B., K.B., T.B., C.R., H.B., P.F., and K.M.; Visualization, A.B.; Supervision, A.B., H.B., P.F., and K.M.; Project Administration, H.B., P.F., and K.M.; Funding Acquisition, H.B., P.F., and K.M. All authors have read and agreed to the published version of the manuscript.

Funding: This study was funded by independent Natural Sciences and Engineering Research Council of Canada's (NSERC) Discovery Grants awarded to H.B., P.F., and K.M. A.B. is funded by NSERC and M.G. DeGroote fellowships.

Acknowledgments: We would like to thank Maarten Voordouw (University of Saskatchewan) for technical and intellectual contributions, and Janet Hill and Champika Fernando (University of Saskatchewan) for help with cpn60 sequencing. We would like to acknowledge the help of Susan Collins (McMaster University) for shipping and receiving samples and reagents. We thank Kathleen Delaney, Dawn Graham, and the staff of the Central Animal Facility at McMaster University for assistance with bat health and care. Multiple students were involved in the collection of samples over the years. The authors would like to acknowledge their help. 
Conflicts of Interest: The authors declare no conflicts of interest.

\section{References}

1. Hayman, D.T. Bats as viral reservoirs. Annu. Rev. Virol. 2016, 3, 77-99. [CrossRef] [PubMed]

2. Stanek, G.; Reiter, M. The expanding Lyme Borrelia complex-Clinical significance of genomic species? Clin. Microbiol. Infect. 2011, 17, 487-493. [CrossRef] [PubMed]

3. Margos, G.; Vollmer, S.A.; Ogden, N.H.; Fish, D. Population genetics, taxonomy, phylogeny and evolution of Borrelia burgdorferi sensu lato. Infect. Genet. Evol. 2011, 11, 1545-1563. [CrossRef] [PubMed]

4. Government_of_Canada. Surveillance of Lyme Disease. Available online: https://www.canada.ca/en/publichealth/services/diseases/lyme-disease/surveillance-lyme-disease.html (accessed on 16 June 2019).

5. Foley, J.; Ott-Conn, C.; Worth, J.; Poulsen, A.; Clifford, D. An Ixodes minor and Borrelia carolinensis enzootic cycle involving a critically endangered Mojave Desert rodent. Ecol. Evol. 2014, 4, 576-581. [CrossRef]

6. Nakao, M.; Miyamoto, K.; Fukunaga, M. Lyme disease spirochetes in Japan: Enzootic transmission cycles in birds, rodents, and Ixodes persulcatus ticks. J. Infect. Dis. 1994, 170, 878-882. [CrossRef]

7. Adeolu, M.; Gupta, R.S. A phylogenomic and molecular marker based proposal for the division of the genus Borrelia into two genera: The emended genus Borrelia containing only the members of the relapsing fever Borrelia, and the genus Borreliella gen. nov. containing the members of the Lyme disease Borrelia (Borrelia burgdorferi sensu lato complex). Antonie Van Leeuwenhoek 2014, 105, 1049-1072. [CrossRef]

8. Reeves, W.K.; Streicker, D.G.; Loftis, A.D.; Dasch, G.A. Serologic survey of Eptesicus fuscus from Georgia, U.S.A. for Rickettsia and Borrelia and laboratory transmission of a Rickettsia by bat ticks. J. Vector Ecol. 2006, 31, 386-389.

9. Socolovschi, C.; Kernif, T.; Raoult, D.; Parola, P. Borrelia, rickettsia, and ehrlichia species in bat ticks, France, 2010. Emerg. Infect. Dis. 2012, 18, 1966-1975. [CrossRef]

10. Michalik, J.; Wodecka, B.; Liberska, J.; Dabert, M.; Postawa, T.; Piksa, K.; Stanczak, J. Diversity of Borrelia burgdorferi sensu lato species in Ixodes ticks (Acari: Ixodidae) associated with cave-dwelling bats from Poland and Romania. Ticks Tick Borne Dis. 2020, 11, 101300. [CrossRef]

11. Piksa, K.; Stanczak, J.; Biernat, B.; Gorz, A.; Nowak-Chmura, M.; Siuda, K. Detection of Borrelia burgdorferi sensu lato and spotted fever group rickettsiae in hard ticks (Acari, Ixodidae) parasitizing bats in Poland. Parasitol. Res. 2016, 115, 1727-1731. [CrossRef]

12. Loftis, A.D.; Gill, J.S.; Schriefer, M.E.; Levin, M.L.; Eremeeva, M.E.; Gilchrist, M.J.; Dasch, G.A. Detection of rickettsia, borrelia, and bartonella in carios kelleyi (Acari: Argasidae). J. Med. Entomol. 2005, 42, 473-480. [PubMed]

13. Haan, C.; Behrmann, I. A cost effective non-commercial ECL-solution for western blot detections yielding strong signals and low background. J. Immunol. Methods 2007, 318, 11-19. [CrossRef] [PubMed]

14. Banerjee, A.; Rapin, N.; Bollinger, T.; Misra, V. Lack of inflammatory gene expression in bats: A unique role for a transcription repressor. Sci. Rep. 2017, 7, 2232. [CrossRef] [PubMed]

15. Links, M.G.; Dumonceaux, T.J.; Hemmingsen, S.M.; Hill, J.E. The chaperonin-60 universal target is a barcode for bacteria that enables de novo assembly of metagenomic sequence data. PLoS ONE 2012, 7, e49755. [CrossRef] [PubMed]

16. Wielinga, P.R.; Gaasenbeek, C.; Fonville, M.; de Boer, A.; de Vries, A.; Dimmers, W.; Akkerhuis Op Jagers, G.; Schouls, L.M.; Borgsteede, F.; van der Giessen, J.W. Longitudinal analysis of tick densities and Borrelia, Anaplasma, and Ehrlichia infections of Ixodes ricinus ticks in different habitat areas in The Netherlands. Appl. Environ. Microbiol. 2006, 72, 7594-7601. [CrossRef] [PubMed]

17. Folmer, O.; Black, M.; Hoeh, W.; Lutz, R.; Vrijenhoek, R. DNA primers for amplification of mitochondrial cytochrome c oxidase subunit I from diverse metazoan invertebrates. Mol. Mar. Biol. Biotechnol. 1994, 3, 294-299.

18. Dibernardo, A.; Cote, T.; Ogden, N.H.; Lindsay, L.R. The prevalence of Borrelia miyamotoi infection, and co-infections with other Borrelia spp. in Ixodes scapularis ticks collected in Canada. Parasit. Vectors 2014, 7, 183. [CrossRef]

19. Kumar, S.; Stecher, G.; Tamura, K. MEGA7: Molecular evolutionary genetics analysis version 7.0 for bigger datasets. Mol. Biol. Evol. 2016, 33, 1870-1874. [CrossRef] 
20. Temmam, S.; Bigot, T.; Chretien, D.; Gondard, M.; Perot, P.; Pommelet, V.; Dufour, E.; Petres, S.; Devillers, E.; Hoem, T.; et al. Insights into the host range, genetic diversity, and geographical distribution of jingmenviruses. mSphere 2019, 4. [CrossRef]

21. Banerjee, A.; Baker, M.L.; Kulcsar, K.; Misra, V.; Plowright, R.; Mossman, K. Novel insights into immune systems of bats. Front. Immunol. 2020, 11. [CrossRef]

22. Netusil, J.; Zakovska, A.; Vostal, K.; Norek, A.; Stanko, M. The occurrence of Borrelia burgdorferi sensu lato in certain ectoparasites (Mesostigmata, Siphonaptera) of Apodemus flavicollis and Myodes glareolus in chosen localities in the Czech Republic. Acta Parasitol. 2013, 58, 337-341. [CrossRef] [PubMed]

(C) 2020 by the authors. Licensee MDPI, Basel, Switzerland. This article is an open access article distributed under the terms and conditions of the Creative Commons Attribution (CC BY) license (http://creativecommons.org/licenses/by/4.0/). 